\title{
A SHARPENED VERSION OF ACZÉL INEQUALITY BY ABSTRACT CONVEXITY
}

\section{RAMAZAN TINAZTEPE* AND GULTEKIN TINAZTEPE}

Abstract. In this study, the Aczél inequality is considered and a new simple proof of the inequality is provided. An extension and a sharper version of this inequality are obtained by performing the results based on the optimality conditions of abstract convex functions.

Mathematics subject classification (2020): 26D07.

Keywords and phrases: Abstract concavity, refinement, Aczél inequality, global optimization.

\section{REFERENCES}

[1] J. ACZÉL, Some general methods in the theory of functional equations in one variable. New applications of functional equations, Uspekhi Mat. Nauk, 11 (3) (1956), 3-68.

[2] G. Adilov, A. M. Rubinov, $\mathbb{B}$-convex sets and functions, Numer. Funct. Anal. Optim., 27 (3-4) (2006), 237-257.

[3] G. Adilov, G. Tinaztepe, The sharpening of some inequalities via abstract convexity, Math. Inequal. Appl., 12 (1) (2009), 33-51.

[4] G. Adilov, I. Yesilce, $\mathbb{B}^{-1}$-convex functions, J. Convex Anal., 24 (2) (2017), 505-517.

[5] K. Chen, F. Yan, H. Y. Yue, Extensions and applications of power-type Aczél-Vasić-Pečarić's inequalities, Math. Probl. Eng., (2019), https://doi.org/10.1155/2019/1323474.

[6] J. L. Díaz-Barrero, M. Grau-Sánchez, P. G. Popescu, Refinements of Aczél, Popoviciu and Bellman's inequalities, Comput. Math. Appl., 56 (9) (2008), 2356-2359, https://doi.org/10.1016/j.camwa.2008.05.013.

[7] G. FArid, J. P. PeČarić, A. U. Rehman, On refinements of Aczél, Popoviciu, Bellman's inequalities and related results, J. Inequal. Appl., 2010 (2010), Article 579567, pages 17, https://doi.org/10.1155/2010/579567.

[8] M. H. HA, Properties and refinements of Aczél type inequalities, J. Inequal. Appl., 12 (1) (2018), 175-189, https://doi.org/10.7153/jmi-2018-12-14.

[9] Z. Hu, A. Xu, Refinements of Aczél and Bellman's inequalities, Comput. Math. Appl., 59 (9) (2010), 3078-3083, https://doi.org/10.1016/j. camwa.2010.02.027.

[10] A. M. Rubinov, Abstract Convexity and Global Optimization, Springer Science Business Media, New York, 2013.

[11] A. M. Rubinov, Z. Y. WU, Optimality conditions in global optimization and their applications, Math. Program., 120 (1) (2009), 101-123.

[12] J. F. TIAN, W. L. WANG, Reversed versions of Aczél-type inequality and Bellman-type inequality, J. Math. Inequal., 9 (2) (2015), 417-424, https://doi .org/10.7153/jmi-09-35.

[13] J. Tian, Y. Sun, New refinements of generalized Aczél inequality, J. Inequal. Appl., 2014 (2014), Article 239, 14 pages.

[14] J. F. TIAN, S. Y. WANG, Refinements of Generalized Aczél's inequality and Bellman's inequality and their applications, J. Appl. Math., 2013 (2013), https ://doi .org/10.1155/2013/645263.

[15] J. Tian, W.-L. WANG, Refinements of Aczél-type inequality and their applications, J. Appl. Math., 2014 (2014), https://doi.org/10.1155/2014/581354.

[16] J. Tian, Y. Zhou, Note on Aczél-type inequality and Bellman-type inequality, J. Nonlinear Sci. Appl., 9 (3) (2016), 1316-1322. 
[17] G. Tinaztepe, S. Kemali, S. Sezer, Z. Eken, The Sharper Form of Brunn-Minkowski Type Inequality for Boxes, Hacet. J. Math. Stat., 50 (2), (2021), 377-386.

[18] S. Vong, On a generalization of Aczél's inequality, Appl. Math. Lett., 24 (8) (2011), 1301-1307, https://doi.org/10.1016/j.aml.2011.02.020.

[19] S. Wu, Improvement of Aczél's inequality and Popoviciu's inequality, J. Math. Inequal., 9 (2007), https://doi.org/10.1155/2007/72173.

[20] W. YANG, Refinements of generalized Aczél-Popoviciu's inequality and Bellman's inequality, Comput. Math. Appl., 59 (11) (2010), 3570-3577, https://doi .org/10.1016/j . camwa. 2010.03.050.

[21] X. Zhou, Some generalizations of Aczél, Bellman's inequalities and related power sums, J. Inequal. Appl., 2012 (2012), Article 130, 11 pages, https ://doi.org/10.1186/1029-242X-2012-130. 